\title{
Trastorno de identidad sexual/transexualidad y salud mental ${ }^{+}$
}

\author{
Mario Souza y Machorro \\ Psiquiatra, psicoterapeuta y psicoanalista. Vocal. Sección Académica de Patología Dual. Asociación Psiquiátrica Mexicana, A.C.
}

\section{RESUMEN}

El trastorno infantil de identidad sexual, que puede o no evolucionar a transexualidad del adulto, presenta condiciones clínicas a vencer que demandan discernimiento clínico a largo plazo. Su sintomatología (insatisfacción que produce un sentimiento persistente de incongruencia con el sexo biológico, un deseo de cambiarlo y de ser conocido desde el punto de vista social y legal en el sexo elegido) y el estado que guarda su grave y poco frecuente condición nosográfica en los estudios internacionales, contrasta su incidencia/prevalencia en los países que la tratan con las diversas teorías sobre su origen. La complejidad del abordaje en el diagnóstico, terapéutica y rehabilitación a largo plazo desde la relativa ausencia de publicaciones en el tema, incluyen la terapia triádica (terapia hormonal para el género deseado, prueba de realidad en el papel deseado y cirugía de reasignación de sexo). Sus alcances en las distintas disciplinas, sus efectos y comorbilidad comprometen distintas áreas de la vida de los pacientes. Dadas las consecuencias irreversibles sobre la salud general de los afectados, se plantean recomendaciones para una cuidadosa visión global que mejore el pronóstico de cada caso a manos de profesionales especializados. Se destacan los derechos de los pacientes a aspirar a una mejor salud y calidad de vida en el marco de la ética de la praxis médico-psicológica. Se insta la participación del punto de vista de las políticas públicas.

Palabras clave: Trastorno de la identidad sexual, transexualidad, diagnóstico, comorbilidad, tratamiento, pronóstico.

\begin{abstract}
Childhood sexual identity disorder, which may or may not evolve into adult transsexuality, presents clinical conditions to overcome that demand long-term clinical discernment. Its symptomatology (dissatisfaction that produces a persistent feeling of incongruence with the biological sex, a desire to change it and to be known socio-legally in the chosen sex) and the state that keeps its serious and infrequent nosoographic condition in international studies, contrast its incidence/prevalence in the countries that deal with it with the various theories about its origin. The complexity of approach in diagnosis, therapeutics and long-term rehabilitation from the relative absence of publications on the subject, include triadic therapy (hormonal therapy for the desired gender, reality test on the desired role and sex reassignment surgery). Its scope in the different disciplines, its effects and comorbidity compromise different areas of patients' lives. Given the irreversible consequences on the general health of those affected, recommendations are made for a careful global vision that improves the prognosis of each case in the hands of specialized professionals. The rights of patients to aspire to better health and quality of life within the framework of the ethics of medical-psychological praxis are highlighted. Participation from the point of view of public policies is encouraged.
\end{abstract}

Keywords: Sexual identity disorder, transsexuality, diagnosis, comorbidity, treatment, prognosis.

Mientras persista un esquema social enajenante, que aliena la consciencia y falsea la realidad, impulsa el uso de drogas, violencia, sexualidad patológica, pobre ponderación de los hechos y nula reflexión, es iluso esperar un desarrollo psicofísico personal, vincular y social saludable.

(Souza y MM. Cannabis y salud: del mito a la evidencia. Alfil. México. 2018)

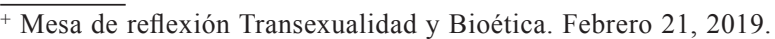
Academia Nacional Mexicana de Bioética.
}

\section{INTRODUCCIÓN}

En el reino animal existen dos sexos: hombre $(\mathrm{H}-\mathrm{B})$ y mujer (M-B). El género, referencia lingüística, alude a: masculino/femenino. Este constructo cultural asume estereotipos sociales asignados a los sexos. ${ }^{1}$ Identidad de género: sentido individual de ser hombre/mujer, a consolidar a los tres o cuatro años de edad. Papel de género: aspectos de conducta individual consistentes con la definición cultural masculinidad/feminidad que devienen en congruente desarrollo de la aceptación como hombre/mujer. Identidad sexual: asunción del individuo como heterosexual, homosexual o bisexual; deriva de lo que ha sido experimentado como eróti- 
camente atractivo y suele consolidarse a partir de la adolescencia. Orientación sexual: atractivo erótico para un individuo, suele ser consistente con la identidad sexual y puede diferir en quien experimenta sentimientos eróticos inaceptables. La Real Academia Española señala: "las palabras tienen género, las personas tienen sexo; es inadmisible decir género como sinónimo de sexo"; para la expresión discriminación o violencia de género la alternativa lingüística es discriminación o violencia por razón de sexo, contra las mujeres, doméstica o de pareja. ${ }^{1}$ Los embriones mamíferos genéticamente hombre/mujer son anatómicamente hembras durante las primeras fases de su vida fetal; su diferenciación la causa el andrógeno fetal entre la sexta y la decimosegunda semana embrionaria. ${ }^{2}$ Los efectos de la masculinización/feminización cerebral se requieren para desarrollar las conductas copulativas y reproductoras de cada sexo. En tal periodo, el feto es vulnerable al suministro exógeno de andrógeno como cuando una madre preñada recibe andrógeno exógeno y causa a su feto femenino el desarrollo de órganos genitales externos similares a los masculinos. ${ }^{3}$ La teoría psicoanalítica añade que la identidad sexual se desarrolla con la formación general de la identidad (fase de separación-individuación) y está influida por la calidad de la relación materna filial. Más tarde (fase edípica previa a la adolescencia), se determina el papel y la orientación sexual. ${ }^{4}$ La Clasificación Internacional de Enfermedades, décima edición, (CIE-10) de la Organización Mundial de la Salud (OMS) incluye el trastorno de la identidad sexual en la infancia (TIS) y el transexualismo (TS) como alteraciones con patrones de comportamiento que causan sufrimiento significativo y desventaja adaptativa. ${ }^{5}$ Su documento CIP-11 que revisa el TS (de aprobarse en la Asamblea Mundial de la Salud en mayo de 2019 entrará en vigor el 1 de enero de 2022), excluirá el TS de la Clasificación Internacional de Enfermedades, undécima edición, (CIE-11), la forma más extrema de la egodistonía sexual, ubicándola como disfunción sexual (DS) para evitar verla como trastorno mental (TM), ya que supone agresión a sus portadores y es causa de discriminación y/o violencia. ${ }^{6}$ No obstante, los clínicos la rechazan como DS porque los trastornos sexuales son en esencia TM. S Saxena, director de Salud Mental de la OMS, señaló: "Queremos que estas personas obtengan ayuda sanitaria cuando la necesiten y agilicen su transición biopsicosocial". ${ }^{6}$

Epidemiología. El TIS/TS se conoce desde 1960; Harry Benjamin de John's Hopkins aportó la cirugía de reconstrucción sexual (CRS) en $1966 .^{7}$ No existe una incidencia/prevalencia precisa del TIS, pero los niños entre nueve y 13 años desisten de ello de 50 a $80 \%,{ }^{8} \sin$ menoscabo de otras alteraciones previas o concomitantes (trastorno de orientación sexual egodistónica (TOSE), comorbilidad física o trastornos adictivos abuso/dependencia por consumo de psicotrópicos (CDPT), ${ }^{9}$ enfermedades de transmisión sexual (ETS) como $\mathrm{VIH}_{1}{ }^{10-15}$ ansiedad, depresión e intentos suicidas). ${ }^{16,17}$ La estadística mundial señala 1:100,000 H-B se "sienten mujer" y 1:200,000 M-B se "sienten hombres", quienes por diversas razones propias y externas, no alterarán su sexo. Informes de especialistas y clínicas de 15 países de Europa observaron en hombres (H-TS) 1:35,000 y en mujeres (M-TS) 1:105,000. Holanda informa cifras más altas de H-TS 1:20,000 y M-TS 1:50,000 atribuidas a contingente extranjero. La estadística europea sugiere $1 \mathrm{H}$-TS en 30,000 y 1 M-TS en 100,000 que afirman desear CRS, aunque no la realicen. ${ }^{18}$

Las consultas infantiles suceden desde los primeros años, pero casi nunca reciben CRS. EI TIS y sus vicisitudes se hace más notorio tras la adolescencia, cuando el paciente decida -o no- corregir lo que llama "error de la naturaleza". ${ }^{19}$ Cerca de 1:30,000 niños y 1:100,000 niñas buscarán en su vida la CRS u optarán por otras acciones. En EUA se estima que $0.3 \%$ de la población es TS. ${ }^{20}$ En Reino Unido, Suecia y Australia la prevalencia varía de 1:50,000 a razón de H-TS de 2.5 a 3-4 por cada M-TS. Pero a diferencia de M-TS adultas (en su mayoría atraídas sexualmente por M-B), los H-TS son atraídos sin distinción por hombres/ mujeres. En muchos países los H-B muestran atracción sexual preferente hacia lo homosexual, H-HS, excepto cuando tienen poco o ningún interés por la actividad sexual priorizando el "ser del sexo complementario". ${ }^{21}$

En otros casos tras la CRS en mujeres, éstas tienen relaciones lésbicas (sin apartarse de su naturaleza masculina). ${ }^{21,22} \mathrm{He}$ ahí la variabilidad de la atipia sexual; de hecho, la literatura sobre TIS/TS muestra una evolución errática al igual que en los imprecisos criterios de clasificación que no siempre se cumplen. ${ }^{20,21,23}$ Años atrás, Lothstein informó la existencia en el mundo de 30,000 casos, $^{21}$ pero sin señalar su proporción con la población de entonces. El Diagnostical Statistical Manual of Mental Disorders, $4^{\text {th }}$ edition, (DSM-IV) estimó prevalencias de $0.005 \%$ a $0.014 \%$ en H-B y de $0.002 \%$ a $0.003 \%$ en $\mathrm{M}-\mathrm{B} .{ }^{23}$ En algunos países se calcula de 1:180 a 1:3,000. ${ }^{21-23}$ En México no hay datos epidemiológicos concluyentes, ${ }^{24}$ el TS y su versatilidad conductual (ya disfuncional y/o parafílica) se recoge como "diversidad sexual", que aspira a normalizar 
todas las modalidades de actividad sexual no naturales a necesidad y capricho de sus portadores.

La comunidad LGBTTTI (lesbianas, "gays", bisexuales, travestis, transexuales, transgénero e intersexuales) ha obtenido mejoras legales y una atención menos discriminativa, pero su atención global sólo dispone de anecdóticos informes de medicina privada. De hecho, de la atención institucional se desconocen: tipo de atención, valoración integral, cuantía, supervisión de la evolución y publicaciones para guiar su manejo. ${ }^{25-28}$ Además, el debate de si el TIS/TS pertenece a los TM o a los físicos (DS) dificulta su abordaje ${ }^{28}$ y omite el trabajo del Dr. Eric Kandel (Premio Nobel de Medicina, 2000) que estableció la inherente dualidad psicofísica de los trastornos sin importar su origen, debido a su ineludible representación mental, lo cual supera el dilema médico etiológico mente-cuerpo en los $\mathrm{TM}^{29}$ por su contexto interactivo de expresión génica/ambiental sobre el desarrollo humano. ${ }^{30}$

Otras controversias del TS son la ética y la estética que posibilitan: asignar tratamiento en ausencia de enfermedad, negar tratamiento a una persona enferma o proveer manejo sólo con fines estéticos. ${ }^{11,23} \mathrm{La}$ mayoría de las personas TIS/TS que muestran o no funcionamiento adecuado en otras áreas de su vida (hay que recordar que ninguna persona es sana a priori) rechazan ser portadoras de TM ignorando su comorbilidad e implicaciones vía racionalización y negación. ${ }^{19}$ Informes señalan que el grupo de H-TS a M-TS tiene mayor edad al momento de solicitar atención e inicia antes manejo hormonal vía automedicación; tiene menor grado educativo y menor cualificación laboral e historia de trastornos adictivos ${ }^{9,18}$ que el de M-TS a H-TS,

Los trastornos sexuales (preocupación de la OMS) no disponen de protocolos de atención, aunque se reconozcan necesarios. ${ }^{21,31}$ Se precisa del sector salud una atención global del TIS/TS y sus consecuencias con guías interdisciplinarias y transdisciplinarias $^{32-37}$ que atiendan, además, su comorbilidad. ${ }^{35}$ Se trata de un TM poco frecuente con impacto personal y social, y abordaje global insuficiente que hace que los pacientes sufran al enfrentarse a muchos obstáculos en la atención a su salud en todo el mundo; sus distintos abordajes muestran resultados variables. ${ }^{35,36}$ En México se desconoce su abordaje integral oficial. ${ }^{24}$

Etiopatogenia. No se conoce en la actualidad. Las hormonas sexuales prenatales definen la conducta sexual típica posterior y la orientación sexual. Los resultados en $\mathrm{H}$-HS y H-TS/M-TS no siendo concluyentes ayudan a entender el TIS por su acción prenatal en el dimorfismo sexual. El sexo biológico, decisivo para determinar la identidad de género, ,33,34 varía en su participación genética, ${ }^{32,33}$ aun en la inamovible dotación XY hombre/XX mujer. ${ }^{3}$ El rechazo de la condición natural por razones biológicas, psicosociales, o ambas, causa grave angustia psicofísica (egodistonía) que llega o no a CRS. ${ }^{31,35-38}$ No existe evidencia de un gen TIS/TS, aunque pueda deberse a variaciones en la secuencia del ADN del gen del receptor de testosterona que altere la variación normal hacia la masculinización del cerebro en $\mathrm{H}-\mathrm{B}$, con sintomatología dada por alteraciones anatomopatológicas (núcleos cerebrales de tamaño intermedio de un área del sector anterior del tálamo, conexiones distintas a lo esperado, etcétera). Otras anomalías genéticas pueden causar trastornos del desarrollo sexual distintos del TIS/TS y condicionar, mas no determinar el TS; ambas, masculinidad/feminidad son estructuras de intensidad variable y expresión fenotípica. ${ }^{32-34}$

La preferencia paterna por un hijo/hija puede afectar también su evolución y su valoración dentro de la familia, aunque tal deseo no es suficiente para influir en el desarrollo de TIS/TS. Evidencia clínica sugiere que para algunas madres de hijos con TIS, cuya incapacidad para elaborar el desacuerdo que les produce el no tener una hija, causa dificultades al relacionarse con él. La capacidad de los padres para moldear la conducta de sus hijos relativa a su género no tiene bases claras, pero consta en la literatura que los padres responden de manera más positiva a las conductas del mismo sexo de sus hijos que a las del otro. La falta de desaprobación paterna a la conducta de género cruzado, un mayor refuerzo de la conducta femenina y menor de la conducta masculina por parte de las madres y la conducta permisiva durante el periodo de consolidación del género podrían interferir con la formación de la identidad del sexo propio. Asimismo, otros factores psicodinámicos promueven la consolidación hacia géneros cruzados respecto a los padres y los constitucionales respecto del niño.

El modelo psicodinámico alude a elementos que aumentan la inseguridad o ansiedad sobre sí mismo que lo hacen vulnerable, que lo llevan a buscar soluciones para reducir su ansiedad (reactividad constitucional al estrés, dificultades tempranas en el establecimiento del apego que incrementan su inseguridad, factores familiares o situacionales que agrandan su ansiedad). En el niño, el nivel de actividad-sensibilidad hace más probable el cruce de género cuando ya ha comenzado a adaptarse a las conductas del sexo complementario, como cuando ocurre en una personalidad no fraguada 
aún, cuyas condiciones apoyan un mecanismo defensivo difícil de declinar de no modificarse los factores participantes de su desarrollo. ${ }^{39-41}$

El origen neurobiológico lo contrastó Landén ${ }^{35}$ con factores psicosociales (papel sexual, pérdidas tempranas infantiles, aspectos de personalidad e intensidad, rigidez y persistencia conductual del otro sexo ${ }^{18,39,40}$ en contextos de sugestión, manipulación, confusión e incluso una especie de "moda" que ocurre en la actualidad). J Money (1970) lo definió como problema de la identidad de género. ${ }^{41} \mathrm{El}$ término disforia de género introducido por N Fisk (1973) fue modificado por Person y Ovesey (1974) al discriminar el transexualismo primario del secundario al enfatizar sus formas mixtas y la dificultad de su distinción. ${ }^{42} \mathrm{R}$ Stoller (1975) diferenció el travestismo del H-HS afeminado (que acepta pertenecer a su sexo biológico y goza sus órganos sexuales). ${ }^{43,44} \mathrm{El}$ término transexual sólo debe asignarse a quienes han pasado por CRS, no a quien lo "desee".

Los factores psicodinámicos sugieren también una intensa simbiosis madre-hijo, de contacto constante que podría inducir comportamiento femenino a causa de la imposibilidad de desvincularse de la identificación de la madre. En las niñas, priva la falta de afecto materno, resentimiento volcado contra su identificación femenina y otras consecuencias. ${ }^{39-41}$ Stoller considera a los H-B portadores de TIS atraídos por hombres de estirpe transgeneracional. ${ }^{42}$ Algunos adolescentes abandonan sus deseos de convertirse en varón y asumen la fachada heterosexual, $\mathrm{H}-\mathrm{HTS}$, pero a nivel inconsciente mantienen el conflicto de la envidia del pene. Las experiencias H-TS iniciales, producen compenetración exagerada con la madre e imprecisión de los límites yoicos, por lo que nunca establecerán una relación H-HTS con ella. Al continuar la relación simbiótica no se desarrollará el conflicto edípico, cuya superación aseguraría su papel H-HTS (identificación con el padre y competencia por la madre). La niña TIS se identifica como niño con el padre y compite con éste por el amor de la madre. ${ }^{39-41}$

Al solicitar la CRS vienen vestidos de la forma que desean ser. La vestimenta femenina identifica un $\mathrm{H}-\mathrm{B}$ : sus ropas llamativas, modales amanerados y su aspecto son notorios; algunos H-TS ya han adoptado nombre del otro sexo y les agrada ser llamados así. Sus caracteres sexuales secundarios (mamas, caderas, formas del cuerpo) son destacados de manera falsa por la automedicación o elementos exógenos. La M-TS que desea ser y sentirse varón, viste como ellos e imita su corte de pelo; en ocasiones los tatuajes pretenden intensificar aún más ese aspecto, disimulan las mamas prominentes con una blusa amplia o una faja apretada en torno al tórax. ${ }^{19}$ Los H-TS/M-TS rechazan los papeles y actividades asignadas a su sexo original por su intenso deseo de vivir en el otro sexo; presentan grave conflicto ante sus sensaciones de hombre/mujer que bloquean el disfrute de sus relaciones. La actividad sexual con el propio sexo tiende a restringirse, no queriendo que les vean o toquen los genitales; sus características sexuales les desagradan por aversión. Los hombres anhelan tener hijos procreados en su vientre y la menstruación la consideran un hecho envidiable; por lo general acuden a orinar sentados y suelen usar ropa interior femenina, además, algunos H-TS realizan ablación peneana por medios propios. ${ }^{19}$

Asimismo, en las niñas se advierte producción excesiva de andrógenos desde la vida intrauterina adquiriendo aspecto varonil. En los hombres la exposición prenatal a hormonas femeninas afecta la expresión del comportamiento masculino convencional. Los niveles anormales de hormonas sexuales antes de nacer y sus efectos conductuales tienden a modificar las experiencias sociales tempranas, sobre todo en actividades, juegos y preferencias atribuibles a su sexo. Se observan niveles bajos de testosterona en H-TS y elevados en M-TS. El tamaño de un área del sector anterior del tálamo podría influir en la orientación sexual. ${ }^{19,20,45-47}$ En suma, no existe explicación clara para el TIS/TS; se cree que influyen factores psicosociales de la primera infancia que parten de condiciones biológicas. ${ }^{41,42}$

Diagnóstico. Un sexo psicológico distinto al somático distingue al sujeto con TIS, éste lo considera equivocado, tratará de asemejarlo y dar apariencia del otro sexo para "eliminar su conflicto"; el nombre TS se prefiere para los adultos. El trastorno presenta alto índice de patología psiquiátrica/física asociada ${ }^{19,20,48}$ y tasa de mortalidad suicida mayor que en la población general. ${ }^{16,17}$ Los TM asociados con mayor frecuencia son: trastornos de personalidad (TP) (narcisista, dependiente y límite), depresión, ansiedad, esquizofrenia, conductas autolesivas o suicidas y trastornos adictivos por CDPT, ${ }^{9,18}$ trastornos sexuales (disfunción [DS] o trastorno de la inclinación sexual [TINS], ETS ${ }^{9-15} \mathrm{u}$ otras. ${ }^{18,47}$ La comorbilidad psiquiátrica es más frecuente en hombres, lo que supone variaciones en la evolución y pronóstico, siendo los TP y la esquizofrenia los asociados a peor evolución, 11,21,34 lo cual invita a su precoz detección, mejoría de la calidad de vida y adherencia al tratamiento. ${ }^{36,49}$ Debe discriminarse la HS, el travestismo, la psicosis y otros trastornos sexuales. El TIS/TS afecta percepción y autoconciencia equívocas 
de ser hombre/mujer, sin afectar las características sexuales biológicas (cromosomas, órganos genitales externos e internos, composición hormonal, gónadas y características sexuales secundarias). La mayoría de ellos inicia en la primera infancia (primario); con menor frecuencia perciben una congruencia inicial entre su identidad sexual y su cuerpo durante muchos años, incluso hasta la adultez (secundario), ${ }^{18,19}$ previa a la inclinación de pertenecer al sexo complementario. Tal convicción puede subsistir estable e inamovible y llegar a afectar el comportamiento así como la atracción sentida, si bien son pocos los casos que llegan a CRS. Estudios electroencefalográficos en H-TS/M-TS indican trazo anormal de 30 a $70 \%$ de ellos, por posible presencia de alteraciones orgánicas cerebrales, independientes de otros TP, físicos y comórbidos. ${ }^{24,31}$

El proceso que finca el manejo debe ser completo: a) Criterios clínicos de la CIE-10 o Diagnostical Statistical Manual of Mental Disorders, $5^{\text {th }}$ edition, (DSM-V) (dada su coincidencia, no sin diferencias). En la CIE-10 (F64.0) Transexualismo (deseo de vivir y ser aceptado como un miembro del género complementario a menudo acompañado del deseo de modificar, mediante métodos hormonales o quirúrgicos, el propio cuerpo para hacerlo lo más congruente posible con el género preferido). A) Identidad transexual persistente al menos por dos años. B) No es síntoma de otro TM ni es secundario a anomalía cromosómica. ${ }^{6}$ En el DSM-V la disforia de género (302.6). Tal incongruencia requiere como mínimo seis meses. A) Se deben cumplir al menos seis de los siguientes criterios manifiestos por deseo intenso de ser del otro sexo e insistencia de serlo: En niños, fuerte tendencia a simular atuendo femenino o travestirse; rechazo a juguetes, juegos, actividades típicamente masculinos y marcada evitación de juegos bruscos. En niñas, resistencia a vestir ropa femenina y usar la masculina; preferencias marcadas y duraderas por el papel del sexo cruzado o fantasías de pertenecer a él; preferencia por juguetes, juegos, o actividades realizadas con compañeros del otro sexo; rechazo a todo lo femenino y disgusto con la propia anatomía. Deseo de poseer los caracteres sexuales primarios y secundarios del sexo deseado. Se asocia a malestar clínicamente significativo o deterioro social, escolar u otras áreas importantes de la vida. Especificar: si existe un trastorno de desarrollo sexual, estado de postransición, vida completa del sexo deseado, recibió o espera tratamiento médico o CRS tipo penectomía, vaginoplastia, mastectomía, o faloplastia. ${ }^{19} \mathrm{La}$ indispensable evaluación médico-psiquiátrica que avala la indicación, comorbilidad, manejo, adecuación, éxito y pronóstico puede contraindicar la CRS. ${ }^{11,47,49}$

Tratamiento. Actividades destinadas al éxito y adaptación a largo plazo: ${ }^{16-18}$ diagnóstico principal y diferencial, identificación de la comorbilidad, criterios de elegibilidad y disposición tipo psicoterapia, prueba de realidad, terapia hormonal, tratamiento quirúrgico. La Harry Benjamin International Gender Dysphoria Association (HBIGDA) indica un equipo interdisciplinario para el diagnóstico y manejo global supervisado a largo plazo. ${ }^{50-57}$ La decisión de CRS es por criterios (diagnóstico de TS, cociente intelectual $(\mathrm{Cl})$ normal, ausencia de patología previa o concomitante, mayoría de edad). Debe cuidarse la posibilidad de error diagnóstico-procedimental con evaluación completa en todos los casos; se contraindica en menores, ante sospecha de psicosis, descompensación o cuando el cambio de sexo es síntoma de otra patología, etcétera. ${ }^{56-61}$ Es útil vincular a los familiares y compañeros sexuales a la evaluación con entrevistas clínicas y pruebas psicológicas que valoran la comorbilidad (TP graves, depresión e intentos suicidas, ansiedad, trastornos alimentarios, estrés postraumático o adaptativo, aversión al sexo, fobia social, DS, TINS y CDPT con trastorno adictivo, autismo, ETS), entre otros. ${ }^{11,62} \mathrm{En}$ caso de rechazo, la devolución de información ayuda a la reconciliación con el sexo H-B/M-B y la promoción del desempeño razonable del papel esperado. La ayuda psicoterapéutica es necesaria en todos los casos. ${ }^{32}$ Ante la imposibilidad de cambio es mejor la aceptación de vivir con el sexo original. ${ }^{11,16,22}$

La cirugía es irreversible, requiere de asumir un genuino nuevo papel y sus consecuencias que incluyen el intento suicida. La prevalencia en quienes no presentaron o negaron sintomatología ${ }^{63-65}$ fue de $41 \%$, cifra mucho más alta frente a $4.6 \%$ de intentos suicidas en la población de EUA e incluso del 10 al 20\% informado en la población LGBTTTI y bisexuales adultos, ${ }^{63-65} \sin$ menoscabo de una mayor morbilidad entre H-TS/M-TS jóvenes que alteraron su sexo y los que no. ${ }^{66}$ La tasa de intento suicida en TS es de 32 a $50 \%$ en todos los países, es inducida por varios factores (discriminación y violencia, rechazo familiar, de amigos y comunidad, acoso por la pareja, policía o público y malos tratos en el sistema de salud; sin considerar la egodistonía (omisión grave) como factor de rechazo). ${ }^{67}$ Un estudio en Irlanda y Reino Unido ${ }^{68}$ (2012) indicó que 78\% de mayores de 18 años mostró ideación suicida; $40 \%$ lo han intentado, $44 \%$ de ellos se autolesionó (38\% en el pasado y $6 \%$ en la actualidad). Pediatrics online (2018) indicó que los adolescentes TS son más propensos al 
intento suicida que aquéllos cuya identidad coincide con el sexo al nacimiento, por lo que se estima que los hombres jóvenes están especialmente en riesgo. En EUA casi la mitad de las personas identificadas como hombre $\mathrm{H}-\mathrm{B}$ asignados al sexo femenino al nacimiento han intentado suicidarse al menos una vez. Casi 30\% de las mujeres TS identificadas como mujeres, pero con certificados de hombre, han intentado suicidarse al menos una vez (cuando menos 28\% de los adolescentes cuestionan su identidad de género). Sin embargo, jóvenes H-TS 10\% y M-TS 18\% han intentado suicidarse pese a que su identidad de género coincide con su certificado de nacimiento. ${ }^{69,70}$

La prevención en H-TS con TP límite u otros TP es el manejo fármaco-psicoterapéutico. I. Prueba de realidad, intenta preparar al H-TS/M-TS a un nuevo y desconocido papel vital; requiere de uno a dos años según sea la condición e incluye cuidado del cabello, cara, voz, ropa, modales a refinar, etc. II. Terapia hormonal, busca obtener la mayor proximidad física al otro sexo. ${ }^{18,52}$ Las características secundarias adquiridas (esteroides) modifican las del propio sexo e inducen otros caracteres sexuales. En los H-TS no hay forma de modificar el esqueleto tras la maduración puberal, ni obtener respuesta total al cambio de vello facial, voz, etcétera. Los estrógenos orales producen ginecomastia (creciente al cabo de dos años de modo variable, atrofia testicular, disminución de la libido y dificultad eréctil; reducción del vello facial y corporal, y disminución del tamaño del pene $)^{54,55}$ y complicaciones (trombosis venosa profunda, trastornos tromboembólicos, hipertensión arterial, aumento de peso, alteración de la tolerancia a la glucosa, alteraciones hepáticas y depresión). ${ }^{53,70} \mathrm{En}$ la M-TS, salvo la genitoplastia, es más fácil obtener características masculinas; los efectos de la testosterona son más intensos que los estrogénicos ${ }^{71}$ (la voz se hará más grave, el clítoris crecerá casi tres veces en el curso de un año, aumento de libido, pilosidad facial y calvicie parcial. Las menstruaciones cesarán en menos de tres a cuatro meses, ${ }^{51,52}$ y en las M-B acné, edema secundario por retención de sodio y deterioro de la función hepática). ${ }^{51,52}$ III. Reasignación sexual, en la M-TS la CRS se efectúa tras manejo hormonal y psicológico satisfactorio de al menos seis meses, sus etapas y técnicas dependen de las características individuales; ${ }^{56}$ el resultado de la genitoplastia masculinizante rara vez interesa totalmente a la paciente, ya que lo deseado es el papel masculino y su conducta, no tanto apreciar las características y funcionalidad del órgano modificado.
La CRS puede facilitar la adaptación psicosocial, beneficiar la relación interpersonal, las manifestaciones psicológicas y el talante, ${ }^{57}$ pero puede haber aflicción grave e inmutable, ${ }^{36,72}$ y efectos comórbidos y/o suicidas. ${ }^{67-69}$ En paciente electivo es un recurso viable no sin riesgos y complicaciones; tras un diagnóstico que limite los factores de arrepentimiento (sucesos infantiles traumáticos, escaso soporte familiar y social, inestabilidad personal, limitadas redes sociales, TP u otra comorbilidad) se valora el vínculo entre orientación sexual y contrición, manifiesto en la orientación de HS con el sexo deseado, mayor, por cierto, en $\mathrm{H}-\mathrm{HTS}{ }^{73}$ La evaluación y manejo integral es individual. ${ }^{18,30,36}$ La incidencia de efectos adversos ocurre por altas dosis y largo tiempo de uso hormonal. ${ }^{74}$ Los H-TS a M-TS tardan dos o más años para CRS e intervención psicológica postquirúrgica (técnicas psicoeducativas, terapia sexológica y abordaje de los diversos problemas del ámbito familiar, laboral, de pareja, etc.). ${ }^{65,66}$ Las M-TS a H-TS reciben mastectomía tras dos a tres años previo a CRS.

Pronóstico. El futuro inmediato y posterior depende de indicadores de resultados positivos, su dimensión y factores estudiados ${ }^{72,73,75}$ (satisfacción quirúrgica, resultado cosmético, calidad de vida, función social, médico psicológica-psiquiátrica). ${ }^{74,76-80}$ Los diversos criterios, instrumentos y metodologías muestran variable utilidad. La predicción de satisfacción postquirúrgica (personalidad estable, apoyo familiar, menor de 30 años, alta motivación y estructura corporal adecuada al nuevo papel) $)^{72}$ se asocia a predictores de mejoría postcirugía (sexo biológico, resultado quirúrgico, diagnóstico adecuado, orientación sexual, mejor función psicosocial previo a la cirugía y apoyo social). ${ }^{73,75} \mathrm{La}$ variedad de conducta sexual previa a CRS (que puede durar muchos años o incluso tras lograrla) muestra que algunos TS cuidan su apariencia de género cruzado e indistinta conducta HS, BS o HTS que indica permanencia en la confusión original no resuelta por reemplazo genito-hormonal; en otros casos la adaptación es adecuada aun sin CRS.

\section{Conclusión}

El desarrollo sano de la persona no abriga dudas sobre su sexo. Los niños entre nueve y 13 años desisten de 50 a $80 \%$, siendo más los casos que nunca llegan a CRS. La evolución TIS-TS, sus vicisitudes y condiciones clínicas reclaman más conocimiento. El cambio genital no garantiza ajuste al otro sexo. De paciente a paciente y de un equipo profesional a otro, existe 
discrepancia de resultados y poca información sobre el grado de mejoría posterior y las razones que impiden llegar a CRS. ${ }^{11,13,53-60}$ Es deseable un adecuado manejo integral estratégico que coordine el nuevo papel y el cuerpo anhelado (desconocido), que aclare la confusión y elimine el conflicto ${ }^{74,77-80}$ en el marco realista de una ética que apoya la productividad y las relaciones sociales. ${ }^{81-84}$ Cabe destacar la importancia de la creación de procesos de información y estadísticas sobre los pacientes H-TS/M-TS para una toma de decisiones informada (incluida la inadecuación al cambio de sexo) y la tipificación de sus problemas derivados, que harán de su registro una valiosa aportación a la salud pública. El sistema nacional de salud no dispone de protocolos para el abordaje de este tema; es preciso abatir el alto costo y consecuencias que resultan de su ausencia.

\section{Bibliografía}

1. Real Academia Española. Diccionario de la lengua española. 23a ed. Madrid, Espasa, 2014.

2. Langman J. Embriología médica. 13ạ ed. Wolters Kluwer. 2016.

3. Kórász K, Simon L. Overview of the biological etiology of transsexualism. Orv Hetil. 2008; 149 (33): 1555-1560.

4. Stoller RJ. Sex and Gender. The transsexual experiment. Hogarth Press. London 1975.

5. OMS. Clasificación de Enfermedades. Capítulo V. Trastornos Mentales, Conductuales y del Desarrollo. Ginebra, 1993.

6. Saxena $\mathrm{S}$ et al. Public health aspects of diagnosis and classification of mental and behavioral disorders: refining the research agenda for DSM-5 and ICD-11 provides a comprehensive. American Psychiatric Pub. 2012. p. 273.

7. Benjamin $\mathrm{H}$. The transsexual phenomenon. New York, Julian Press. 1966.

8. Steensma T, Biemond R, de Boer F et al. Desisting and persisting gender dysphoria after childhood: a qualitative follow-up study. Clin Child Psychol Psych. 2011; 16: 499-516.

9. Souza y Machorro M. Adicciones visión integral de su problemática, abordaje y terapéutica: Patología Dual. Facultad de Medicina, UNAM. Editorial Prado, México, 2014.

10. Movilh. Disponible en: http://www.movilh.cl/historico-la-oms-dejade-considerar-a-la-transexualidad-como-una-enfermedad-mental/.

11. Souza y Machorro M. Trastornos adictivos y sexuales (DS, TIS, TINS, TOS, TOSE) en HLBT. Patología Dual. Editorial Prado, México, 2019.

12. American Psychiatric Association. DSM-5. Diagnostic and statistical manual of mental disorders. DSM-5. Arlington, VA, American Psychiatric Association, 2014.

13. Gates GJ. How many people are lesbian, gay, bisexual, and transgender? The Williams Institute. 2011. Available in: http:// williamsinstitute.law.ucla.edu/wp-content /uploads/ GatesHow-Many-People-LGBT-Apr-2011.pdf.

14. APA. American Psychiatric Association. Diagnostic and statistical manual of mental disorders. DSM-IV-TR. Washington, DC. 2000.

15. Souza y Machorro M. Sexualidad femenina. Trillas. Universidad Autónoma del Estado de Hidalgo-Feria Universitaria del Libro, FUL. México. Agosto 24, 2018.
16. Garaizabal C. Evaluación y consideraciones psicológicas. En: Gómez E, Esteva I. (eds.) Ser transexual. Barcelona, Glosa, 2006.

17. Esteva de Antonio I et al. Evaluación endocrinológica y tratamiento hormonal de la transexualidad en la Unidad de Trastornos de Identidad de Género en Andalucía de Málaga. Cirugía Plástica Ibero-latinoamericana. 2001: 27 (4): 273-280.

18. Brown GR. Trastornos de la identidad sexual y transexualidad. 2019. www.msdmanuals.com /es-mx/hogar/trastornos-de-lasalud-mental/sexualidad/trastornos-de-la-identidad-sexual-ytransexualidad. 2019 Merck Sharp \& Dohme corp. Kenilworth, NJ., USA.

19. Lothstein L. The postsurgical transsexual: empirical and theoretical considerations. Arch Sex Behav. 1980; 9 (6): 547564.

20. Martí MG. Trastornos de la identidad sexual. En: AL Montejo González. Sexualidad y salud mental. Editorial Glosa, Barcelona, 2007.

21. Das A, Wilhelm M, Prakriti T. People of the third sex: understanding homosexuality, transgender identity and intersex conditions in Hinduism, Filadelfia, Xlibris Corp, 2004.

22. Cohen-Reteñís PT, Owen A, Káiser VG et al. Demographic characteristics, social competence, and behavior problems in children with gender identity disorder: a cross-national, crossclinic comparative analysis. J Abnorm Child Psychol. 2003; 31 : 41-53.

23. Jiménez LJL, Maldonado GME, Jiménez MN. Protocolo de atención para pacientes con disforia de género. Arch Neurocien. 2017; 22 (1): 35-40.

24. Bergero T, Cano G et al. La transexualidad: asistencia multidisciplinar en el sistema público. Rev Asoc Esp Neuropsiq. 2004; 24: 2.828-2.838.

25. Acuerdo A/023/2010 del C. Procurador General de Justicia del DF, que crea la Unidad Especializada para la Atención a Usuarios de la Comunidad LGBTTTI. Disponible en: http:// www. cgservicios.df.gob.mx/prontuario/vigente/r379 601.doc.

26. Salin-Pascual RJ. Aportaciones para la comprensión de las personas transexuales. Rev Mex Neuroci. 2007; 8 (6): 575-585.

27. Protocolo de atención sanitaria a personas transexuales. Servicio Canario de Salud. Gobierno de Canarias, España. Disponible en: http://www2.gobiernodecanarias. org/sanidad/scs.

28. Kandel ER. Biology and the future of psychoanalysis: a new intellectual framework for psychiatry. Am J Psychiatry. 1999; 156: 505-524.

29. Hofer MA; Sullivan RM. Toward a Neurobiology of Attachment. In: Nelson CA, Luciana M (eds). Handbook of Developmental Cognitive Neuroscience. MIT Press, 2008.

30. Smith E, Junger J, Derntl B, Habel U. The transsexual brain: a review of findings on the neural basis of transsexualism. Neurosci Biobehav Rev. 2015; 59: 251-266.

31. Gómez E, Esteva I et al. Ser transexual. Dirigido al paciente, a su familia, y al entorno sanitario, judicial y social, Barcelona, Glosa, 2006.

32. Chung WC, Auger AP. Gender differences in neurodevelopment and epigenetics. Pflugers Arch. 2013; 465: 573-584.

33. Souza y MM. Utilidad de la psicoterapia y psicoanálisis en patología dual: complementariedad terapéutica. Revista APM Psiquiatría. 2014; 2 (2): 30-44.

34. World Health Organization. Developing sexual health programmers: a framework for action. Available in: http:// whqlibdoc.who.int/hq/2010/WHO_RHR_HRP_10.22_eng. pdfl.

35. Landén M, Wålinder J, Lunström. Prevalence, incidence and sex ratio of transsexualism. Acta Psychiatr Scand. 1996; 93: 221-223. 
36. Landén M et al. Factors predictive of regret in sex reassignment. Acta Psychiatr Scand. 1998; 97 (4): 284-289.

37. Good L, Byron J. Medicina, racionalidad y experiencia. Una perspectiva antropológica. Barcelona, Bellaterra, 2003.

38. Sellipi T. ¿De qué hablamos cuando hablamos de factores en salud? En: Comelles J, Perdiguero E et al. Medicina y cultura. Estudios sobre Antropología y Medicina, Barcelona, Bellaterra, 2000, pp. 249-275.

39. Person E, Ovesey L. The transexual syndrome in males. I Primary transsexualism. Am J Psychotherapy. 1974; 28 (1): 4-20.

40. Stoller RJ. Sex and Gender, New York, Science House, 1968.

41. López MN, Calleja CM. Transexualidad: una alteración cerebral que comienza a conocerse. Cuadernos de Bioética. 2016; 27: 81-94.

42. Ingalhalikar M, Smith A, Parker D, Satterthwaite TD, Elliott MA et al. Sex differences in the structural connectome of the human brain. Proc Natl Acad Sci USA. 2014; 111: 823-828.

43. Gizewski R, Krause E, Schlamann M, Happich F et al. Specific cerebral activation due to visual erotic stimuli in male-to-female transsexuals compared with male and female controls: an fMRI study. J Sex Med. 2009; 6: 440-448.

44. Souza y Machorro M. Dinámica y evolución de la vida en pareja. Psique conflicto y vínculo erótico-sexual. EAE Editorial Academia Española. 2012.

45. Martín CA. Antropología del género. Culturas, mitos y estereotipos sexuales. Madrid, Cátedra, 2006.

46. Moore H. Antropología y feminismo, Madrid, Cátedra, 1999.

47. Gooren L. El transexualismo, una forma de intersexo. En: Becerra-Fernández A. Transexualidad. La búsqueda de una identidad, Madrid, Díaz de Santos, 2003.

48. Agencia de Evaluación de Tecnologías Sanitarias de Andalucía, Consejería de Salud. Junta de Andalucía, Informe preliminar: Problemática de salud ligada a la transexualidad en Andalucía. Posibilidades de abordaje e inclusión entre las prestaciones del sistema sanitario público de Andalucía, Mayo de 1999.

49. Imperato-McGinley J, Peterson RE, Gautier T, Sturla E. Androgens and the evolution of male-gender identity among male pseudo-hermaphrodites with 5alpha-reductase deficiency. N Engl J Med. 1979; 300 (22): 1233-1237.

50. Saro Cl. Transexualidad: una perspectiva transdisciplinaria. México: Alfil. 2009.

51. HBIGDA. The Harry Benjamin International Gender Dysphoria Association. The standards of care for gender identity disorders. 6th version, Minneapolis, 2001.

52. Seal LJ. A review of the physical and metabolic effects of crosssex hormonal therapy in the treatment of gender dysphoria. Ann Clin Biochem. 2016; 53: 10.20.

53. Unger A. Hormone therapy \& transgender patients. Transl Androl Urol. 2016; 5: 877-884.

54. Frade M, Bilharinho B. Clinical management of transsexual subjects. Arq Bras Endocrinol Metab. 2014, São Paulo Mar. 2014; 58 (2): 188-196. http://dx.doi.org/10.1590/00042730000003091.

55. Hembree WC, Cohen-Kettenis P, Delemarre-van de Waal HA, Gooren LJ et al. Endocrine treatment of transsexual persons: an endocrine society clinical practice guideline. J Clin Endocrinol Metab. 2009; 94 (9): 3132-3154.

56. Martin den Heijer M, Bakker M, Gooren L. Long term hormonal treatment for transgender people. BMJ. 2017; 359: j5027.

57. Berli JU, Knudson G, Fraser L et al. What surgeons need to know about gender confirmation surgery when providing care for transgender individuals. JAMA Surg. 2017; 152: 394-400.
58. Colebunders B, Brondeel S, D'Arpa S, Hoebeke P et al. Update on the surgical treatment for transgender patients. Sex Med Rev. 2017; 5 (1): 103-109.

59. Montejo GAL. Esquizofrenia y sexualidad. Editorial. Rev Enferm Salud Ment. 2017; 6: 3-4.

60. Sadock BJ, Sadock VA, Ruiz PM. Esquizofrenia y sexualidad. Editorial. Rev Enferm Salud Ment. 2017. 11th. Walters Kluwer, 2015.

61. Cohen P, Van Gooren S. Sex reassignment of adolescent transsexuals: a follow-up study. J Am Acad Child Adolesc Psychiatry. 1997; 36 (2): 263-276.

62. Souza y MM. Alcohol y personalidad. Patología Dual. Alfil. México, 2018.

63. Fitzgibbons R. Gender dysphoria, gender identity disorder and informed consent. 2015. Available in: http://www.childhealing. com/articles/genderidentitydisorder.php.

64. Reisner S, Vetters R, Leclerc M et al. Mental health of transgender youth in care at an adolescent urban community health center: a matched retrospective cohort study. J Adolesc Health. 2015; 56: 274-279.

65. Dhejne C, Lichtenstein P et al. Long-term follow-up of transsexual persons undergoing sex reassignment surgery: cohort study in Sweden. PLoS One. 2011; 6 (2): e16885.

66. De Vries AL; Noens IL; Cohen-Kettenis PT. et al. Autism spectrum disorders in gender dysphoric children and adolescents. J Autism Dev Disord. 2010; 40 (8): 930-936.

67. Simonsen RK, Giraldi A et al. Long-term follow-up of individuals undergoing sex reassignment surgery: psychiatric morbidity and mortality. Nord J Psychiatry. 2016; 70 (4): 241-247.

68. Rapaport L. Trans teens much more likely to attempt suicide. Pediatrics, [On Line]. 2018. Available in: www.reuters.com/ article /us-health-transgender-teen-suicide/trans-teens-much -more-likely-to-attempt-suicide-idUS- KCN1LS39K.

69. Virupaksha HG, Muralidhar D, Ramakrishna J. Suicide and suicidal behavior among transgender persons. Indian J Psychol Med. 2016; 38 (6): 505-509.

70. Transgender Equality Network Ireland 2019. Suicide in Trans Communities. New Data from the Mental Health \& Well-being Survey. Trans Mental Health \& Well-being Survey: Self-harm \& Suicide.www.teni.ie/suicide_in_trans communities_new_data_from_the_mental_heälth_well ${ }_{-}^{-}$ being_survey.

71. Chan W, Drummond A et al. Deep vein thrombosis in a transgender woman. CMAJ. 2017; 189: E502-E504.

72. Irwig MS. Testosterone therapy for transgender men. Lancet Diabetes Endocrinol. 2017; 5: 301-311.

73. Fabris B, Trombetta C, Belgrano E. II Transessualismo: identificazione di un percorso diagnostico e terapéutico, Milano, Kurtis Editrice, 1999.

74. Lindemalm G, Körlin D, Uddenberg N. Prognostic factors vs. outcome in male-to-female transsexualism. A follow-up study of 13 cases. Acta Psychiatr Scand. 1987; 75 (3): 268-274. https:// doi.org/10.1111/j.1600-0447.1987.tb02788.x.

75. Blanchard R et al. Pronostic factors of in postoperative transsexuals. Can J Psychiatry. 1989; 34 (1): 43-45.

76. Hess J, Rossi RN, Panic L, Rübben H, Senf W. Satisfaction with male-to-female gender reassignment surgery. Dtsch Arztebl Int. 2014; 111 (47): 795-801.

77. Asscheman H, Gooren L. Gender dysphoria: interdisciplinary approaches in clinical management. New York, Bockting W, Coleman E. eds. Haworth Press, 1992.

78. Bodlund O, Armelius K. Self-image and personality traits in gender identity disorders: an empirical study. J Sex Marital Ther. 1994; 20 (4): 303-317. 
79. Bodlund O, Kullgren G. Transsexualism: general outcome and pronostic factors: a five-year follow-up study of 19 transsexuals in the process of changing sex. Arch Sex Behav. 1996; 25 (3): 303-3016.

80. Van Kesteren $\mathrm{P}$ et al. Mortality and morbidity in transsexual subjects treated with cross-sex hormones. Clin Endocrinol. 1997; 47 (3): 337-342.

81. Briggs PC, Hayes S, Changaris M. Somatic experiencing informed therapeutic group for the care and treatment of biopsychosocial effects upon a gender diverse identity. Front Psychiatry. 2018; 9: 53.

82. Hurtado F, Gómez M, Donat F. Transexualismo y salud mental. Revista de Psicopatología y Psicología Clínica. 2007; 12 (1): 43-57.
83. Hembree WC, Cohen-Kettenis PT, Gooren L et al. Endocrine treatment of gender-dysphoric/gender-incongruent persons: an endocrine society clinical practice guideline. J Clin Endocrinol Metab. 2017; 102: 3869-3903.

84. Tollinche LE, Burrows WC, Radix A, Long M et al. Perioperative care of the transgender patient. Anesth Analg. 2018; 127 (2): 359-366.

Correspondencia:

\section{Mario Souza y Machorro}

Cristóbal Colón 75-15. Col. Tlalcoligia. Tlalpan 14430.

Ciudad de México.

E-mail: souzaym@yahoo.com 